\title{
User Centered Ecological Interface Design (UCEID): A Novel Method Applied to the Problem of Safe and User-Friendly Interaction Between Drivers and Autonomous Vehicles
}

\author{
Kirsten Revell ${ }^{1(\bowtie)}$, Pat Langdon ${ }^{2}$, Mike Bradley², Ioannis Politis ${ }^{2}$, \\ James Brown ${ }^{1}$, and Neville Stanton ${ }^{1}$ \\ ${ }^{1}$ Human Factors Engineering, Transportation Research Group, University of Southampton, \\ Building 176, Boldrewood Innovation Campus, Burgess Road, Southampton, SO16 7QF, UK \\ \{K.M.Revell, J.W.Brown, Neville.Stanton\} @Soton. ac.uk \\ 2 Department of Engineering, Engineering Design Centre, University of Cambridge, \\ Trumpington Street, Cambridge, CB2 1PZ, UK \\ pm124@eng.cam.ac.uk, \{mab54,ip332\}@cam.ac.uk
}

\begin{abstract}
User Centered Ecological Interface Design (UCEID) is a novel Human Factors method that integrates relationships between Ecological Interface Design (EID) and inclusive Human Centered Design. It combines existing methodology from the Cognitive Work Analysis (CWA) framework [1-3] and Inclusive User Centered Design $[4,5]$. This paper offers a practical guide to UCEID by providing a high-level summary for practitioners using the example of vehicle to driver handover in a BASt Level 3 autonomous vehicle.
\end{abstract}

Keywords: Human Factors methods · Human-systems integration Autonomous vehicles · User Centered Design · Cognitive Work Analysis · EID Inclusive design

\section{Introduction}

Human Factor (HF) methods exist to tackle problems relating to the interaction between human and other elements of the system, that existing methods of design, evaluation and procurement have failed to address. These types of problems are often resistant to purely technical interventions resulting in less effective system performance [6]. With ever increasing rates of technological advancement, it is more difficult for companies to compete on functionality, reliability or cost [7]. Human Factors methods offer a means to provide a competitive edge by harnessing technology to enable people to accomplish meaningful, real-world tasks.

Human Factors methods fall into range of categories that are relevant for application at different parts of the design process [6]. The UCEID process includes a combination of 'Data Collection', 'Task Analysis' and 'Cognitive Task Analysis' techniques. Figure 1 shows how different methods are suitable at different stages of the design 
process. The UCEID method is positioned early in the design process to allow 'analytical prototyping', the means of applying HF insights to systems or designs that are yet to exist in physical form. It covers a combination of 'Identify need' and 'Develop Concept' stages of the design process taking the analyst to 'initial design concept' stage, not final concept. Key finding from inclusive User Centered Design advise an active process of linked iteration between technology prototypes and user trials is necessary to meet the dual needs of diverse user demographics and technology delivery requirements [8] (see Fig. 1).

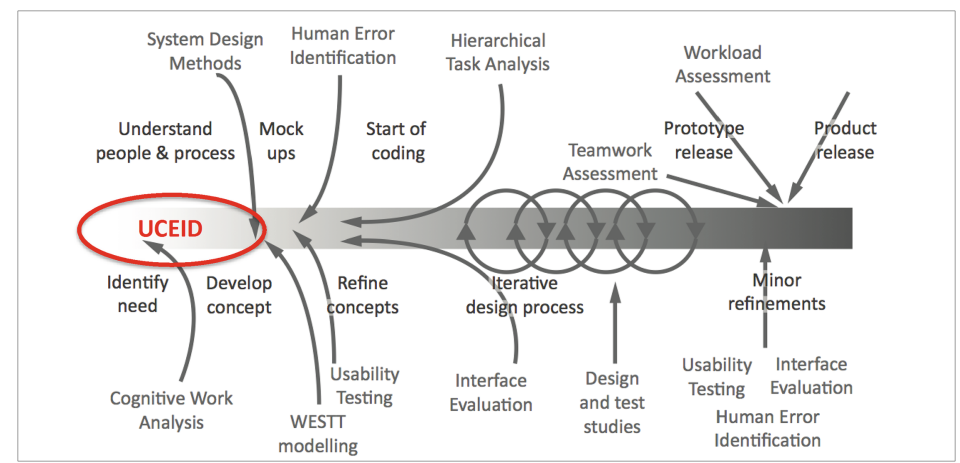

Fig. 1. Diagram to show where the UCEID method fits into the design process in relation to other HF methods. Amended from Stanton et al. (2013).

Ecological Interface Design (EID) is based on Gibsonian methodology that aims to make constraints of the system and environment explicit, so that the appropriate action is apparent to the system user [9]. UCEID is a novel HF method that integrates relationships between EID and inclusive Human Centered Design by combining existing methodology from the Cognitive Work Analysis framework [1-3] and Inclusive User Centered Design $[4,5]$. The approach engages with stakeholders, Subject Matter Experts (SMEs) and users to produce outputs that generate design requirements. Initial design concepts are then produced following a design workshop and concept filtering activity. This paper presents a high-level summary of the steps within UCEID, applied to vehicle to driver handover in a BASt Level 3 autonomous vehicle.

\section{The UCEID Method}

This section follows the structure recommended for Human Factors (HF) methods [6] that provides a process flow chart followed by a generic description of each step with selected examples. The UCEID method is best suited to complex sociotechnical systems where the user plays a critical role in the interaction. Domains that are complex exude some of all the following qualities: high risk, dynamic, uncertain, with interconnected parts. Vehicle initiated vehicle to driver (VtoD) handover in a BASt Level 3 autonomous vehicle fits the criteria of a complex sociotechnical system and will be used to illustrate application of the method. 


\subsection{Flowchart}

The flowchart in Fig. 2 describes the sequence of steps (rectangles) for the full UCEID method. Steps within boxes can be undertaken in parallel. Decision points (diamonds) and feedback loops occur at different points in the process (Fig. 2).

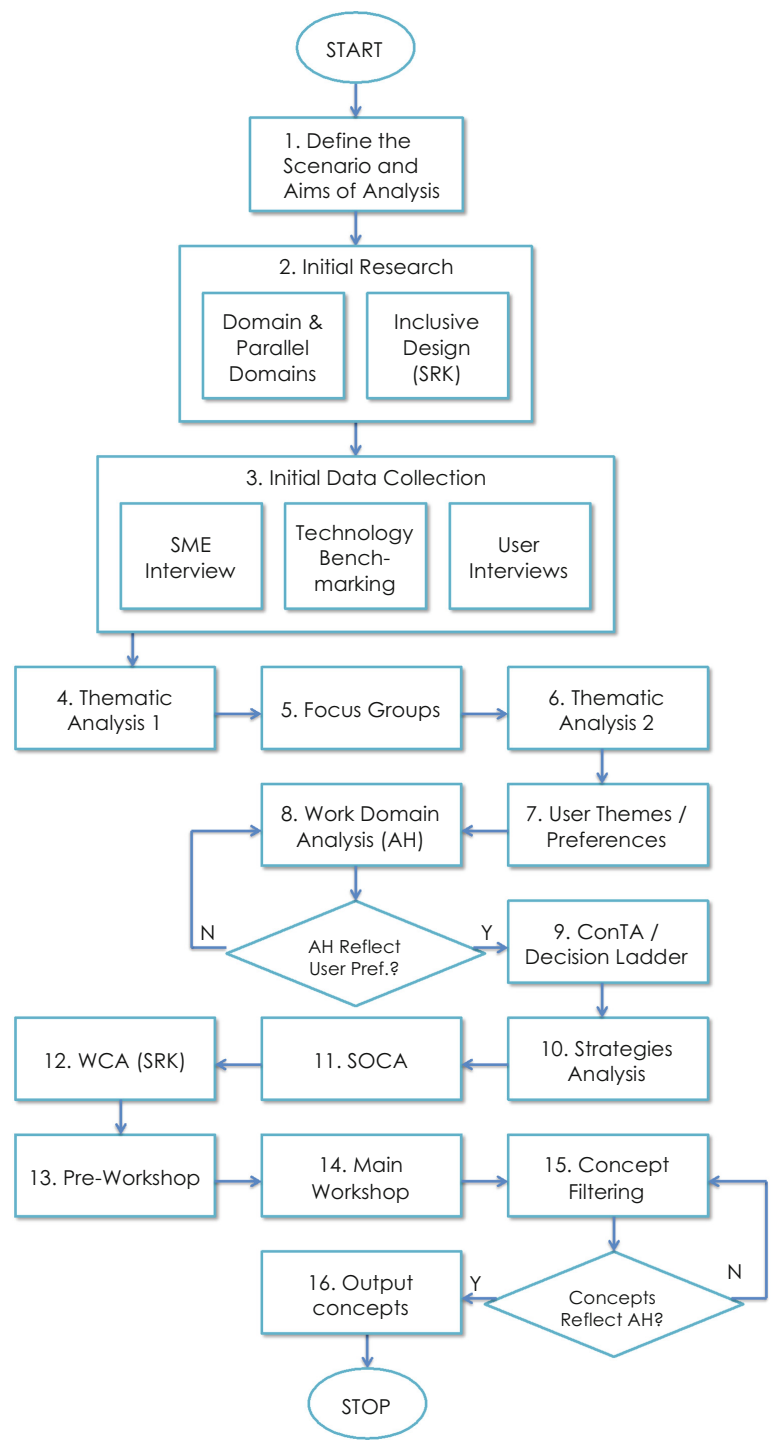

Fig. 2. Flowchart showing the sequence of steps for the prototype UCEID method 


\subsection{Procedures and Advice}

This section describes the different steps in the UCEID method (depicted in Fig. 2) using examples from the autonomous vehicles domain where appropriate.

Step 1 - Define the Scenario, Aims and Boundaries of Analysis. The aims of analysis, target scenario and boundaries of analysis need to be clearly defined. For example: analysis of 'transfer of control in autonomous vehicles' for the scenario 'planned transfer of control from vehicle to driver on a highway in a BASt level 3 vehicle'. Of the CWA elements (8-12), steps $8 \& 9$ fulfill the EID requirements [9] but other CWA steps are optional depending on focus and resources.

Step 2 - Initial Research. Different members of a research group can undertake 'Researching domain \& parallel domains' (e.g. transfer of control in the aviation domain [10] and requirements of advanced driving [11]) and 'Inclusive design' (e.g. reference to literature and national surveys relating to user needs for automation [12]), in parallel to scope the research.

Step 3 - Initial Data Collection. Similarly, initial data collection can occur in parallel. For the chosen scenario, 'SME semi-structured interviews' would occur with automation experts. 'Technology analysis and benchmarking' would be used to understand current commercial offerings and relevant technologies relating to autonomous vehicles. 'User interviews' would occur with target users of autonomous vehicles following standard approaches $[13,14]$.

Step 4 - Thematic Analysis 1. Using a grounded theory approach [13] data from Step 3 should be thematically analyzed. Qualitative software such as NVIVO can assist with this step. Generated themes should be defined and compared with independent analysts to create a final set. Themes relating to autonomous vehicles included 'Trust', 'Control' and 'Safety'.

Step 5 - Focus Groups. Focus groups representing an inclusive range of the target users is recommended for generalizable outputs (e.g. driving population with balanced gender mix and range of legal driving age groups, from variety of geographical regions). Two researchers (a moderator and facilitator) and a set of 5-7 participants per group are suggested.

Step 6 - Thematic Analysis 2. Data from previous steps are consolidated and triangulated according to the data source [15]. The most robust data is tabulated to capture requirements for step 7 .

Step 7 - Preferences User Themes Interpreted. This step takes themes from focus groups and interviews and converts them into insights (e.g. 'auditory alert of increasing urgency' when handover is required, as users 'anticipate being otherwise occupied' during automated mode). 
Step 8 - Work Domain Analysis (WDA) Abstraction Hierarchy (AH). This step marks the initial phase of the CWA framework. WDA describes the constraints that govern the purpose and function of the systems under analysis. The output of this step is the creation of an $\mathrm{AH}[1,2]$. This is a diagram constructed of 5 levels of abstraction, from the most abstract level 'purpose' (e.g. planned transfer of control back to driver), to 'values' (e.g. safety), 'purpose related functions' (e.g. ensure driver situation awareness), 'functional purpose' (e.g. communicated vehicle status to driver) to the most concrete form, 'physical object' (e.g. head up displays).

Step 9 - Control Task Analysis (ConTA). ConTA looks in depth at constraints that are imposed by tasks that need to be carried out in specific situations to reveal not only the constraints, but the level of flexibility in how activities can be achieved [16]. For autonomous vehicles, three time periods were used to constrain the scenario (e.g. Prehandover, Handover \& Post-handover).

Step 10 - Strategies Analysis. This analysis is useful for exploring the flexibility within a system for different types of strategies within different contexts [2,17]. The strategy adopted by an agent in a particular situation may vary based on workload, or vary between agents (e.g. different strategies for different user groups (e.g. elderly versus young drivers) or the same user undergoing different journeys (e.g. work or leisure).

Step 11 - Social Organisation and Cooperation Analysis (SOCA). SOCA identifies how control tasks can be distributed between human and non-human agents within the system [17]. It determines how social (e.g. driver) and technical (e.g. automation) aspects of a system can work together to enhances performance as a whole. SOCA reveals the flexibility of system to deal with unanticipated events.

Step 12 - Worker Competencies Analysis (WCA). WCA is the final CWA element and involves identifying the cognitive skills required for control task performance in the system under analysis. These are classified using the Skills, Rules and Knowledge (SRK) framework [18].

Step 13 - Pre-workshop. Following new insights from steps 9 onwards, the pre-workshop allows re-calibration of the scenario (Step 1) user preferences (Step 7) and AH (Step 8). This enables a clear and consistent presentation of the context and design tasks to participants in the Design Workshop (Step 14). The basis for concept filtering (Step $15)$ is defined here.

Step 14 - Design Workshop. The workshop is used to shares and review the knowledge gained from previous steps about the problem and solution spaces, then to generate concept solutions to solve that problem (e.g. handover interaction designs, alerts or alarms, multimodal variations). Inclusive design process should be adopted to explore needs and create solutions.

Step 15 - Concept Filtering. Ideas and concepts from the workshop are transcribed and a design criterion matrix [19] relating to the AH in step 8 is constructed. Design 
features are rated according to inclusion and adherence to the criteria to produce candidate 'output concepts'.

Step 16 - Concept Development. Final output concepts are created through an iterative process applied to the candidate concepts. Interface Analysis Methods are applied to assess their viability, and Design Methods are used to create an initial version of the concepts [6]. The Output Concepts should be documented with sufficient detail to enable their implementation and evaluation in later stages of the design process, in order to arrive at the final design.

\section{Summary}

A high-level overview of UCEID, a new method incorporating EID and Inclusive User Centred Design principles has been presented to help HF practitioners. Examples relating to VtoD handover in a BASt Level 3 autonomous vehicle have been provided.

Acknowledgments. This work was supported by Jaguar Land Rover and the UK-EPSRC grant EP/011899/1 as part of the jointly funded Towards Autonomy: Smart and Connected Control (TASCC) Programme.

\section{References}

1. Rasmussen, J., Pejtersen, A., Goodstein, L.P.: Cognitive Systems Engineering. Wiley, New York (1994)

2. Vicente, K.J.: Cognitive Work Analysis: Toward Safe, Productive, and Healthy ComputerBased Work. Lawrence Erlbaum Associates, Mahwah (1999)

3. Jenkins, D.P., Stanton, N.A., Salmon, P.A., Walker, G.H.: Cognitive Work Analysis: Coping with Complexity. Ashgate Publishing Ltd., Farnham (2009)

4. Czajkowski, K., Fitzgerald, S., Foster, I., Kesselman, C.: Grid information services for distributed resource sharing. In: 10th IEEE International Symposium on High Performance Distributed Computing, pp. 181-184. IEEE Press, New York (2001)

5. Langdon, P., Thimbleby, H.: Inclusion and interaction: designing interaction for inclusive populations. Editor. Spec. Ed. Interact. Comput. 22, 439-448 (2010)

6. Stanton, N.A., Salmon, P.M., Rafferty, L.A., Walker, G.H., Baber, C., Jenkins, D.P.: Human Factors Methods: A Practical Guide for Engineering and Design. Ashgate Publishing Ltd., Aldershot (2013)

7. Green, W.S., Jordan, P.W.: Human Factors in Product Design: Current Practice and Future Trends. Taylor \& Francis, London (1999)

8. Langdon, P.M., Lazar, J., Heylighen, A., Dong, H. (eds.): Inclusive Designing: Joining Usability, Accessibility, and Inclusion. Springer, Heidelberg (2014)

9. McIlroy, R.C., Stanton, N.A.: Ecological interface design two decades on: whatever happened to the SRK taxonomy? IEEE Trans. Hum-Mach. Syst. 45(2), 145-163 (2015)

10. Handover/Takeover of Operational ATC Working Positions/Responses. https:// www.skybrary.aero/index.php/Handover/Takeover_of_Operational_ATC_Working_Positions/ Responses 
11. Roadcraft: the police driver and rider handbooks for better, safer driving. http:// www.roadcraft.co.uk

12. IM Travellers needs Survey (2015). https://ts.catapult.org.uk/current-projects/travellerneeds-uk-capability-study/

13. Glaser, B.G., Strauss, A.L.: The Discovery of Grounded Theory: Strategies for Qualitative Research. Aldine, Chicago (1967)

14. Breakwell, G.M.: Introducing Research Methods in Psychology. Research Methods in Psychology. Sage, London (2000)

15. Ewe, F.: An Introduction to Qualitative Research, 3rd edn. Sage, London (2006)

16. Naikar, N., Moylan, A., Pearce, B.: Analysing activity in complex systems with cognitive work analysis: concepts, guidelines, and case study for control task analysis. Theor. Issues Ergon. Sci. 7(4), 371-394 (2006)

17. Naikar, N.: An examination of the key concepts of the five phases of cognitive work analysis with examples from a familiar system. In: Proceedings of the Human Factors and Ergnomics Society $50^{\text {th }}$ Annual Meeting, pp. 447-451 (2006)

18. Rasmussen, J.: The role of hierarchical knowledge representation in decision making and system management. IEEE Trans. Syst. Man Cybern. 15, 234-243 (1985)

19. Pugh, S.: Total Design: Integrated Methods for Successful Product Engineering. Addison, Wokingham (1993) 